\title{
Sparfloxacin-induced photo-onycholysis and photosensitivity characteristically sparing lepromatous skin lesions: an interesting observation
}

\author{
Manoj Pawar ${ }^{1 凶}$, Mehak Singh ${ }^{2}$
}

\begin{abstract}
Sparfloxacin is an antibiotic in the quinolone group of antibacterial agents, which often induce photosensitive skin reactions, more often phototoxic reactions than photoallergic ones, and sometimes associated photo-onycholysis. We present a case of phototoxic dermatitis with photo-onycholysis in a 38-year-old man probably induced by sparfloxacin, which was prescribed to him along with rifampicin and clofazimine because he was suffering from borderline lepromatous leprosy. He developed exaggerated sunburn-like eruptions mainly on sun-exposed sites along with painful onycholysis of the fingernails. Interestingly, the hypopigmented patches of leprosy were spared, which is a very rare phenomenon. Withdrawal of sparfloxacin along with administration of systemic steroids and other supportive measures helped heal the skin eruptions with hyperpigmentation, but the photo-onycholysis was slow to resolve.
\end{abstract}

Keywords: sparfloxacin, photosensitivity, photo-onycholysis, sparing, leprosy

Received: 27 August 2017 | Returned for modification: 22 September 2017 | Accepted: 12 October 2017

\section{Introduction}

Fluoroquinolones are a recognized but uncommon cause of photosensitive dermatitis. Sparfloxacin (SPFX), a fluoroquinolone, has a wide range of antibacterial activity and is often a secondline drug for treatment of leprosy $(1-3)$. We report a case of phototoxic dermatitis that spared lepromatous skin lesions with photoonycholysis probably induced by SPFX.

\section{Case report}

A 38-year-old Indian male with a dark complexion (Fitzpatrick Type V) and a diagnosed case of borderline lepromatous (BL) leprosy presented with a sunburn-like eruption on the face, upper back, extremities, and trunk of 2 weeks' duration (Figs. 1-4). It was associated with spontaneous fingernail discoloration (Fig. 5). The nails showed a color change from yellow to brownish-black with mild pain. The rash first appeared on sun-exposed parts (the face, extensors of the arm, and upper back) and later involved other body parts but characteristically spared covered parts of the body and BL skin lesions. There was a history of photo-aggravation of the lesions. Starting 2 months earlier, he was being treated with multidrug therapy for multibacillary leprosy (i.e., MDT-MB; dapsone, rifampicin, and clofazimine) according to WHO guidelines by a dermatologist. Six weeks after starting this treatment, the patient suffered from "Dapsone syndrome"; hence it was stopped and substituted with oral SPFX $400 \mathrm{mg} /$ day. One week later, the patient experienced the symptoms above, and so he consulted us. Cutaneous examination showed intense, diffuse erythema mainly over sun-exposed parts with islands of spared BL leprosy patches; in places, desquamation of the skin was present. All of the fingernails showed irregular yellowish discoloration and separation of the distal half of the nail plate. The toenails were unaffected. Thorough general examination of the patient including hair and mucous membranes was insignificant. All relevant laboratory in- vestigations, including antinuclear antibody titer, porphyrin levels, $\mathrm{KOH}$ examination, and bacterial and fungal cultures of the nails, were normal or negative.

In view of SPFX-induced photodermatitis, that treatment was stopped and we prescribed ofloxacin in tablet form, $200 \mathrm{mg}$ b.i.d. Tapering doses of oral prednisolone with antihistamines and strict photoprotective measures including broad-spectrum sunscreens were given. The patient's skin lesions improved significantly and healed with hyperpigmentation by a follow-up visit 2 weeks later, whereas the nail lesions were slow to resolve.

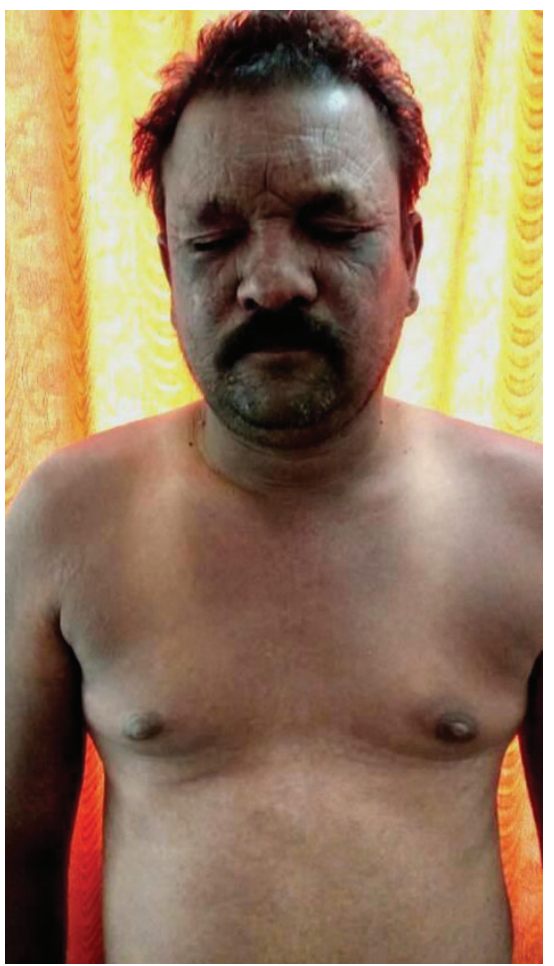

Figure 1 | Sunburn-like eruption sparing covered parts of the body (vest) and desquamation on the face. 


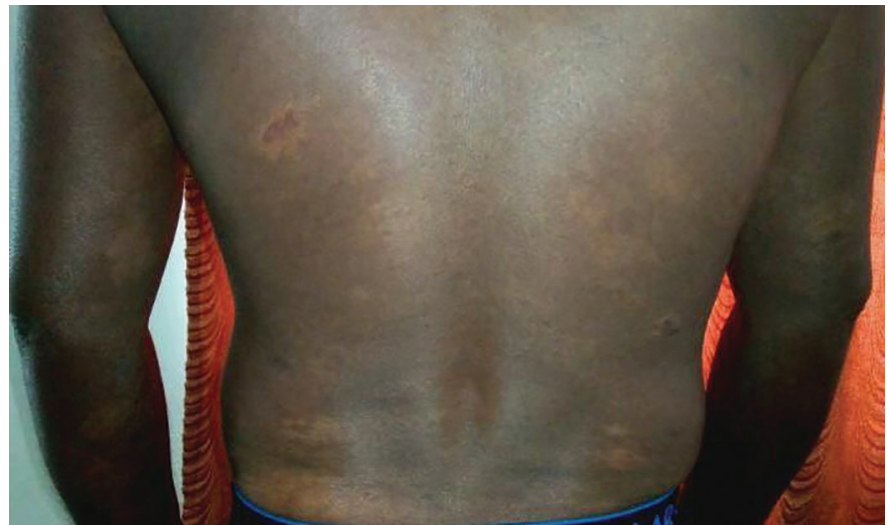

Figure 2 | Sunburn-like reaction over the trunk and arms, sparing hypopigmented macules of borderline lepromatous leprosy on the trunk.

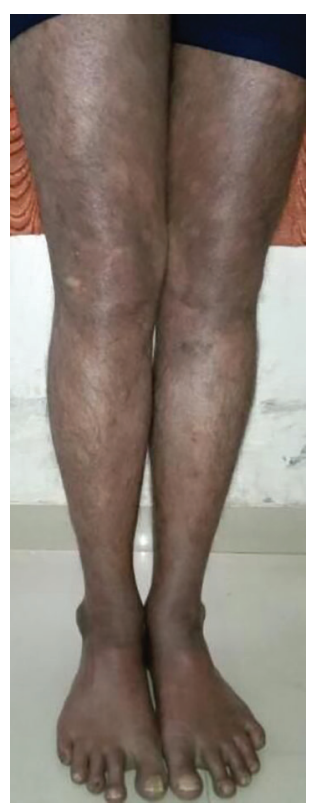

Figure 3 | Similar lesions sparing hypopigmented patches of borderline lepromatous leprosy on the legs.

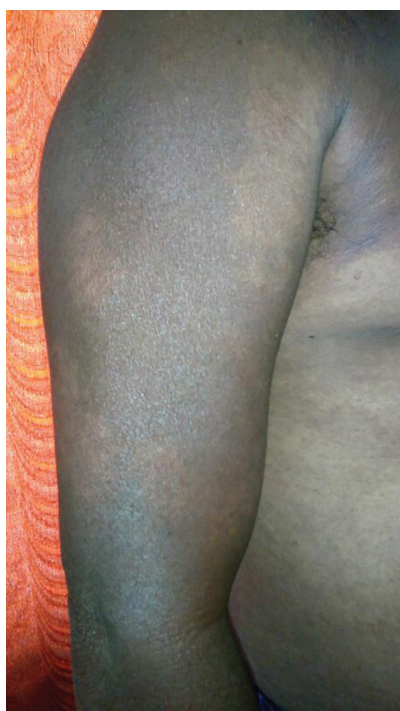

Figure 4 | Similar lesions sparing hypopigmented patches of borderline lepromatous leprosy on the arm.

\section{Discussion}

SPFX is a difluorinated quinolone antimicrobial agent used for Gram-positive, Gram-negative, and anaerobic infections. Common adverse reactions noted during treatment with SPFX are

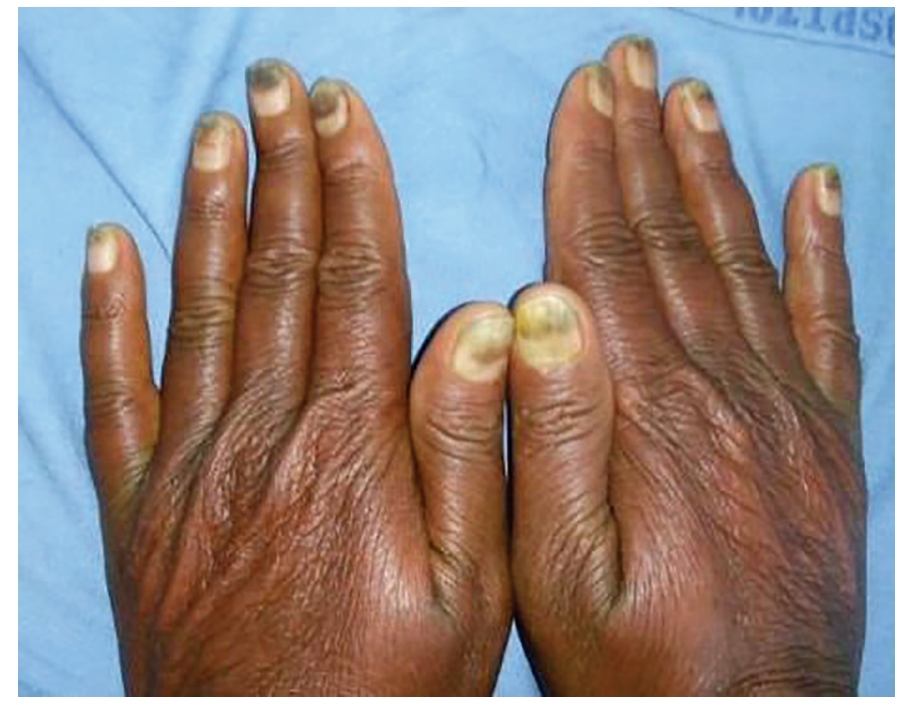

Figure 5 | Photo-onycholysis of all fingers showing muddy brownish-yellow dis coloration on the distal half.

gastrointestinal disturbances, headache, and phototoxic reactions, the symptoms of which appear minutes to hours after sun exposure and often peak after a few hours to several days. The overall incidence of SPFX-induced phototoxicity is about 8\% (1). When used at a higher dosage, SPFX often causes photosensitive dermatitis with a typical ashy gray color over the malar region after about 5 to 7 days, and it sometimes induces a lichenoid tissue reaction with prolonged exposure (2-4). The photosensitivity reaction may be sunburn-like, eczematous, lichenoid, or sometimes bullous (2). The phototoxicity of SPFX is unique because photosensitive dermatitis is due to a direct UV-dependent photodynamic phototoxic effect of the SPFX and it is evoked by photoaugmentation between UVA and UVB (4). SPFX accumulates inside the melanin of melanocytes and keratinocytes, increasing its tissue concentration in the basal epidermis, thus leading to long-lasting photoinjury and liquefaction degeneration of the basal cells (5). Mahajan et al. (6) have proposed that the photosensitivity of SPFX is dose dependent because it is more commonly observed at a dose of $400 \mathrm{mg} /$ day rather than $200 \mathrm{mg} /$ day, whereas Dawe et al. (7) have observed that a dosage of SPFX at $200 \mathrm{mg} /$ day was much more photoactive. However, this variation in phototoxic dosage may be due to differences in the skin types of the patients studied in the reports.

Photo-onycholysis induced by SPFX is often distal, half-moon shaped, and surrounded by pigmentation. It may accompany but often follows a cutaneous photosensitivity reaction. A possible mechanism of photo-onycholysis is that the lack of melanin and absence of sebum and granular layer in the nail favors the penetration of UV radiation (8). The spectrum of photo-onycholysis is in the UVA range, whereas UVB fails to reach the nail bed (9). Four distinct types of photo-onycholysis have been described depending on their appearance. Our patient developed Type I photo-onycholysis because all of the fingers were involved with halfmoon-shaped separations of the distal nail plate. Type II affects a single finger and exhibits a well-defined circular notch opening distally with a proximal brownish hue. Type III, which involves the central part of the nail bed of several fingers, is defined as round yellow stains of the nail that turn red after 5 to 10 days. Type IV has been associated with bullae under the nails, mostly caused by tetracycline hydrochloride (10).

Interestingly, the hypopigmented patches of BL leprosy were spared, producing an "islands of white in a sea of red" appear- 
ance in our patient. Sparing of a Hansen's patch in a drug rash has previously been described in dapsone and ampicillin hypersensitivity $(11,12)$. A possible explanation of this phenomenon is reduced release of vasoactive amines and reduced substance $\mathrm{P}-$ mediated immunostimulation and cellular proliferation, which results from reduced neuropeptide release because of nerve damage in lepromatous skin lesions (11).

Monochromator phototesting is the gold standard for testing systemic drug photosensitivity (13); unfortunately, we could not perform it in our patient because he was lost to further follow-up. However, onycholysis, sunburn-like skin reaction after the very first dose of SPFX, and its temporal association is highly suggestive of SPFX phototoxicity rather than photoallergy in our case. Moreover, photoallergy has not been reported yet with SPFX (2).

Patients with SPFX-induced photosensitivity should be treated as early as possible, preferably within 2 weeks of onset, to prevent lichenoid tissue reaction (14). Avoidance of direct sunlight and of the offending drug are the mainstays of treatment. Protective clothing and a broad-brimmed hat, appropriate UVA and UVB

\section{References}

1. Matsumoto N, Akimoto A, Kawashima H, Kim S. Comparative study of skin phototoxicity with three drugs by an in vivo mouse model. J Toxicol Sci. 2010;35:97100.

2. Vassileva S, Mateev G, Parish LC. Antimicrobial photosensitive reactions. Arch Intern Med. 1998;158:1993-2000.

3. Mahajan VK, Sharma NL. Photo-onycholysis due to sparfloxacin. Australas J Dermatol. 2005;46:104-5.

4. Tokura Y, Iwamoto Y, Mizutani K, Takigawa M. Sparfloxacin phototoxicity: potential photoaugmentation by ultraviolet A and B sources. Arch Dermatol Res. 1996;288:45-50.

5. Hamanaka H, Mizutani H, Asahig K, Shimizu M. Melanocyte melanin augments sparfloxacin-induced phototoxicity. J Dermatol Sci. 1999;21:27-33.

6. Mahajan BB, Gupta RR, Garg GJ. Sparfloxacin-induced photosensitivity - dose related. Indian J Dermatol Venereol Leprol. 2000;66:276.

7. Dawe RS, Ibbotson SH, Sanderson JB, Thomson EM, Ferguson J. A randomized controlled trial (volunteer study) of sitafloxacin, enoxacin, levofloxacin and sparfloxacin phototoxicity. Br J Dermatol. 2003;149:1232-41.

8. Baran R, Juhlin L. Drug-induced photo-onycholysis. Three subtypes identified in a study of 15 cases. J Am Acad Dermatol. 1987;17:1012-6. sunscreens, and an evening dosing strategy can minimize the risk of photosensitivity. Topical steroids combined with soothing lotions reduce the local inflammatory response and pruritus, and systemic antihistamines and steroids may be needed in severe cases.

Our case closely resembles a case studied by Mahajan et al. (3) because that patient was an Indian male with Fitzpatrick Type $\mathrm{V}$ skin and photodermatitis with photo-onycholysis was observed in both cases, but our patient's lepromatous skin lesions were characteristically spared whereas Mahajan et al.'s patient suffered from pulmonary tuberculosis and did not exhibit such a phenomenon.

\section{Conclusion}

SPFX-induced photosensitivity and photo-onycholysis should be readily recognized by dermatologists so that it can be appropriately investigated and promptly treated to avoid serious sequelae such as lichenoid reaction, which is very difficult to treat.
9. Logan RA, Hawk JL. Spontaneous photo-onycholysis. Br J Dermatol. 1985;113: 605-10.

10. Hanneken S, Wessendorf U, Neumann NJ. Photodynamic onycholysis: first report of photo-onycholysis after photodynamic therapy. Clin Exp Dermatol. 2008;33: 659-60.

11. Gopinath H, Nayak K, Kumar P, Chakraborti S. Spared white islands in a drug rash: revisiting sparing phenomenon in Hansen's disease. Int J Dermatol. 2017; 56:e102-4.

12. Pavithran K. Sparing of leprosy macule in ampicillin hypersensitivity rash. Indian J Lepr. 1987;59:309-12.

13. Khandpur S, Porter RM, Boulton SJ, Anstey A. Drug-induced photosensitivity: new insights into pathomechanisms and clinical variation through basic and applied science. Br J Dermatol. 2017;176:902-9.

14. Hamanaka H, Mizutani H, Shimizu M. Sparfloxacin-induced photosensitivity and the occurrence of a lichenoid tissue reaction after prolonged exposure. J Am Acad Dermatol. 1998;38:945-9. 